\title{
La lengua de las inscripciones métricas laconias anteriores al 400 a.C. ${ }^{1}$
}

\author{
Paloma GuiJarRo RuANO \\ Universidad Complutense de Madrid \\ paloma.guijarro@hotmail.com
}

Recibido: $14-01-2014$

Aceptado: 16-06-2014

\section{RESUMEN}

En este trabajo se analiza la lengua que presentan las inscripciones laconias en verso anteriores al ca. 400 a.C. Nuestro objetivo es determinar si en estos textos se reproduce fundamentalmente el modelo jónico o si, por el contrario, se emplea el dialecto local. Tras examinar los rasgos dialectales que comparten los epigramas laconios con las inscripciones en prosa de la misma región, por un lado, y con el dialecto épico, por otro, llegamos a la conclusión de que, contrariamente a la opinión extendida, la poesía epigráfica laconia se compone en dialecto epicórico y que no evita los rasgos locales más marcados de este.

Palabras clave: epigrama, dialecto, lengua, épica, Laconia.

\begin{abstract}
In this paper we analyse the language in which metrical inscriptions from Laconia prior to $c .400 \mathrm{BCE}$ are written. More specifically, we try to find if the linguistic Ionic model is mainly reproduced or if, on the contrary, a local dialect is used. Once considered the dialect features shared by Laconian epigrams, prose inscriptions from the same region and epic dialect, we conclude that, contrary to a widespread view, they are written in the epichoric dialect and that its local features are not avoided.
\end{abstract}

Keywords: Epigram, dialect, language, Epic, Laconia.

\section{INTRODUCCIÓN}

Antes del periodo helenístico no se habían definido aún las convenciones del género epigramático por lo que la composición de inscripciones en verso no estaba asociada todavía a un dialecto (literario) o a un metro concreto. Con el paso del tiempo estos

\footnotetext{
${ }^{1}$ Este trabajo ha sido realizado dentro del proyecto de investigación Contactos interdialectales en griego antiguo. Estudio a partir de los textos epigráficos (FFI 2012-35721-C02). Agradezco a Alcorac Alonso Déniz así como a los informantes anónimos sus útiles y pertinentes comentarios sobre la versión preliminar del texto. Todas las imprecisiones y errores que pueda contener se deben únicamente a la autora.
} 
textos fueron perdiendo su finalidad epigráfica hasta que a partir del s. IV a.C. nació el epigrama literario como tal con unas características formales más definidas. ${ }^{2}$

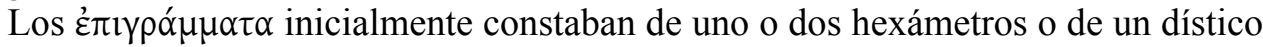
elegíaco. Estaban destinados a ser inscritos sobre un objeto y su función venía determinada por su contenido. Al componerse en el mismo verso que la épica y la elegía, empleaban habitualmente expresiones atestiguadas en estos dos grandes géneros, pero presentaban también formas ajenas al jonio literario.

\section{LA LENGUA DE LAS INSCRIPCIONES EN VERSO}

Existen diferentes estudios que se han ocupado de analizar las inscripciones métricas desde un punto de vista lingüístico. Su objetivo es determinar en qué variedad(es) dialectal(es) están compuestas -en algún dialecto literario o en un dialecto epicórico-y explicar, cuando interviene más de un dialecto, cómo se produce la interacción entre ellas. El contacto lingüístico se da sobre todo entre la lengua de la épica (y/o de la elegía), en tanto que variedad de prestigio y modelo de composición, y el dialecto local, más ligado al habla cotidiana de los poetas y de los grabadores del texto. Mientras que en época arcaica y clásica sólo estaban bien estandarizadas las lenguas literarias — sobre todo las de la épica—, no existió una variedad epigráfica de carácter suprarregional hasta la llegada de la koiné jónico-ática.

\subsection{Hipótesis sobre la lengua de los epigramas ${ }^{3}$}

Esencialmente, se han planteado dos hipótesis para explicar la mezcla de rasgos literarios y epicóricos en la poesía epigráfica. Para autores como Buck (1924), los epigramas se componían fundamentalmente en el jónico de la épica, aunque luego se les podía dar un barniz dialectal. A medida que se retrocede en el tiempo, el elemento local está más presente y, cuanto más se avanza, su lengua se asimila más al jonio de la épica hasta su progresiva imposición en la composición epigramática posterior. ${ }^{4}$ En cambio, para otros estudiosos los diferentes dialectos epicóricos constituyen la base lingüística de estos textos. Dentro de este grupo Mickey (1981a, b) concluye que los poetas empleaban una variedad suavizada del dialecto local que excluía el empleo de las formas dialectales más marcadas, mientras que según Kock (1910) y Alonso Déniz y Nieto Izquierdo (2009) componían en su propio dialecto sin evitar los rasgos epicóricos más característicos. ${ }^{5}$

${ }^{2}$ Para un estudio de los términos con que se designan los epigramas (epigráficos y literarios) en el mundo griego, cf. Puelma (1997). Para la evolución del epigrama posterior, cf. Bing y Bruss (2007).

${ }^{3}$ Para una revisión de las principales hipótesis, cf. Alonso Déniz y Nieto Izquierdo (2009: 84-89).

${ }^{4}$ Cf. Thumb y Kieckers (1932: 221 ss.) o Hoffmann, Debruner y Scherer (1973: 109-113).

${ }^{5}$ Kretschmer (1915: 275), en línea con Kock (1910) y contradiciendo a Buck (1912: 351 ss.), defendió como si se tratara de una regla lingüística que los epigramas se componían en el dialecto del poeta y que estos únicamente recurrían a las formas jónicas por motivos métricos. Posteriormente, Buck (1923: 133 ss.) a la hora de replicar la hipótesis de Kretschmer, le dio el sobrenombre de «ley de Kretschmer». 
El principal defecto de estos trabajos es el estudio parcial de los datos. ${ }^{6}$ Asimismo, muchos de ellos parten de hipótesis lingüísticas preconcebidas que condicionan los resultados. ${ }^{7}$ Tampoco explican los factores que pudieron condicionar la elección de la forma literaria o de la epicórica cuanto estas eran métricamente equivalentes. De todas las aproximaciones metodológicas al estudio de la lengua de las inscripciones métricas, la de Alonso Déniz y Nieto Izquierdo (2009) aplicada a los textos de la Argólide parece la más objetiva debido a que parte del examen directo y exhaustivo de los datos sin ningún condicionamiento lingüístico previo para después clasificar sus rasgos en función de si son exclusivos de los epigramas, compartidos con la prosa dialectal o con la lengua del epos.

\section{OBJETO Y MÉTODO DE NUESTRO ESTUDIO ${ }^{8}$}

El corpus objeto de estudio está formado por 15 inscripciones métricas procedentes de Laconia anteriores al siglo III a.C., ${ }^{9}$ fecha en que se detecta la aparición de la koiné jónico-ática en la zona. Se han incluido SEG 46. 400 (Esparta, ca. 400-375 a.C.) y CEG 1.821 (Esparta, ¿s. IV a.C. in.?) por ser los primeros carmina en alfabeto reformado ya que contribuyen a la interpretación de algunas grafías epicóricas ambiguas.

Tras un estudio lingüístico previo de estos textos, se han establecido dos niveles de análisis. En primer lugar, se ha aplicado al material laconio la metodología propuesta por Alonso Déniz y Nieto Izquierdo (2009), de manera que los rasgos dialectales de las inscripciones en verso se han clasificado atendiendo a si son compartidos con la prosa laconia o con la poesía épica (\$4). Se pretende determinar así su grado de dependencia respecto de la tradición epigráfica local o de la lengua del epos y comparar así los resultados obtenidos con los de la vecina Argólide. En un segundo momento, para determinar si esos rasgos se emplean en un contexto de equivalencia métrica, se han clasificado atendiendo al criterio de organicidad que Tiberi (1996: 71-85) empleó

${ }^{6}$ Cf. Buck (1912, 1924), Thumb-Kieckers (op.cit.) o Kretschmer (op.cit.). Aunque Mickey (1981a) examinó todo el corpus de inscripciones métricas tesalias, pretendió que sus conclusiones fueran extrapolables al resto de poesía epigráfica.

${ }^{7}$ Mickey (1981b) se basa en una preselección de rasgos lingüísticos que considera representativos de diferentes dialectos literarios y epicóricos, si bien su aparición u omisión en los textos puede ser simplemente fruto del azar.

${ }^{8}$ No se han estudiado las versiones literarias de las inscripciones métricas porque en los epigramas literarios se ha producido un proceso de normalización lingüística consistente en adaptar las formas epicóricas originales al dialecto jónico. Sobre esta cuestión, cf. Palumbo (1987: 429-434), Tiberi (1996: 71-85), y Kaczko (2009: 90-117). Sirva como ejemplo la versión de Paus. (5. 24. 3) del laconio $C E G$ 1.367 (Laconia < Olimpia, ca. 490-480 a.C.).

${ }_{9} S E G$ 26, 457, Laconia, ca. 675-650 a.C.; CEG 1.368, Giteo, ¿ca. 600-500 a.C.?; CEG 1.369, Delfos < Laconia, ¿ca. 600-550 a.C.?; CEG 1.370, Delfos < Laconia, ¿s. vi?; $C E G$ 1.371, Olimpia < Laconia, ¿ca. 550-525 a.C.?; $C E G$ 1.372, Olimpia < Laconia, ¿ca. 550-525 a.C.?; CEG 1.373, Selasia, ca. 525 a.C.; $C E G$ 1.374, Esparta, ¿ca. 530-500 a.C.?; $C E G$ 1.375, Esparta, ¿ca. 530-500 a.C.?; CEG 1.376, Esparta, ¿ca. 510-500?; CEG 1.377, Esparta, ¿ca. 500-475 a.C.?; CEG 1.378, Esparta, ca. ¿450?-431; CEG 1.367, Olimpia < Laconia, ca. 490-480 a.C.; SEG 46. 400, Esparta, ca. $400-375$ a.C. y CEG 2.821, Esparta, ¿s. IV a.C. in.? 
para los epigramas literarios: un rasgo es orgánico cuando su escansión es diferente según se emplee la forma dialectal o la epicórica, pero inorgánico cuando no se da una oposición métrica porque ambas son métricamente equivalentes (\$5).

\section{RASGOS DIALECTALES DE LAS INSCRIPCIONES MÉTRICAS LACONIAS ANTERIORES A CA. 400 A.C.}

Son pocos los rasgos lingüísticos comunes a la epigrafía laconia (en verso y en prosa) y a los poemas homéricos debido a las diferencias tan marcadas entre el dialecto epicórico y el de la épica; ${ }^{10}$ en cambio, son más numerosos los compartidos por la poesía epigráfica sólo con la prosa laconia contemporánea (§4.1) o con Homero (§4.2). Incluso, algunos rasgos dialectales se han conservado únicamente por el testimonio de las inscripciones en verso ( $\$ 4.3)$.

\subsection{Rasgos compartidos por las inscripciones en verso y en prosa}

\subsubsection{El vocalismo}

Además de los abundantes ejemplos de conservación de /a:/ esperables en las inscripciones métricas laconias, ${ }^{11}$ la forma $T \imath v \delta \alpha \rho \imath \delta \tilde{\alpha} v$ de $C E G 1.373$ (Selasia, ca. 525 a.C.) «de los Dioscuros» es característica del dialecto epicórico frente al más común

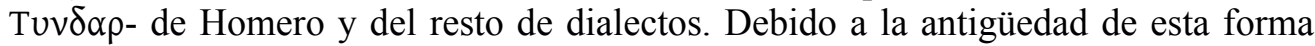
métrica así como a otros correlatos posteriores, ${ }^{12}$ lo más probable es que Tiv $\delta \alpha \rho^{\circ}$ sea el vocalismo local, probablemente secundario respecto de Tuv $\delta \alpha \rho^{\circ}$.

También es dialectal el teónimo 'A $\operatorname{c} \lambda(\lambda) \bar{v} v$ de $C E G 1.370$ (Delfos < Laconia, s. vI a.C.), cuyo timbre /e/ anterior a la forma con asimilación ('A $\pi$ ó $\lambda \lambda \omega v$ ) se mantuvo incluso en los textos laconios compuestos en koiné. ${ }^{13}$ Sin embargo, a pesar de que el término se corresponde con la fonética local, presenta un alargamiento artificial en la primera sílaba que debe atribuirse a la lengua del epos (§4.2.2).

Las inscripciones en verso y en prosa presentan los mismos resultados para las contracciones vocálicas. El resultado esperable en /a:/ $(</ \mathrm{a}: /+/ \mathrm{o}(:) /)$ se testimonia

${ }^{10}$ vid. pronombre tol (cf. $C E G$ 1.371, Laconia < Olimpia, ¿ca. 550-525 a.C.?), el vocalismo $/ \mathrm{a} /<*_{8}$,

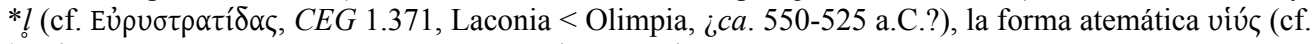

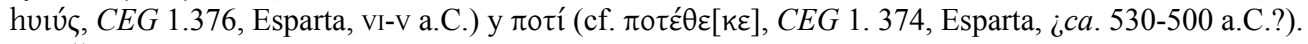

${ }^{11}$ El primer caso de $<\mathrm{H}>(<* / \mathrm{a}: /)$ es del s. IV (CEG 1.822, Gerontras, ¿s. IV a.C.?) aunque en el texto coexiste con $<\mathrm{A}>$ y contrasta con otros cinco carmina del mismo periodo (SEG 11. 654; SEG 46. 400; $C E G 2 \mathrm{nn} .819-821)$ que sólo presentan $<\mathrm{A}>$. Sólo resulta problemático hı $\lambda$ हैFō[1] (CEG 2.367, Laconia

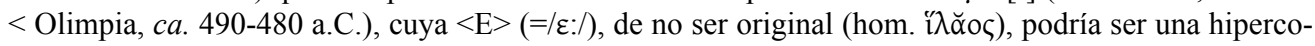
rección debida a un lapicida eleo, cf. Striano (1989: 34).

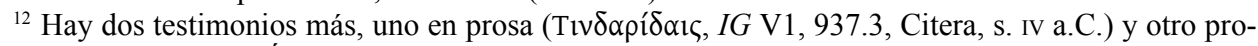
cedente del santuario de Ártemis Ortia (Tı $\delta \alpha \rho 1 \delta \tilde{\alpha} v, I G$ V1, 305.5-6, s. II d.C.) cuyo material epigráfico, aunque tardío, apunta a una revitalización del dialecto local por oposición a la koiné (Alonso Déniz 2014). A partir del s. III a.C. se generalizó $<$ Y $>$ en la región (Striano 1989: 29-32 §11.142).

${ }^{13}$ Cf. Striano (1989: 18-20). 
en el genitivo singular [ ]oí $\delta \bar{\alpha}$ ( $C E G$ 1.376, Esparta, ¿ca. 510-500 a.C.?), en el plural

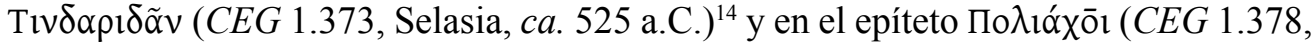

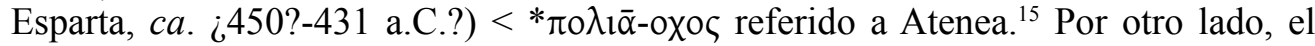
resultado dialectal de /a/ + /e:/ se atestigua en hop ̃̃v (CEG 2.821, Esparta, IV a.C.).

En cuanto a las contracciones isovocálicas, $/ \mathrm{a}: /(<* \theta \bar{\alpha} \mathrm{F} \bar{\alpha})$ se da en $\sigma \tilde{\alpha} \sigma \theta \alpha \mathrm{l}$ y $\sigma \tilde{\alpha} \tau \rho \circ$ (SEG 46. 400, Esparta, 400- 375 a.C.); ${ }^{16}$ los resultados de /e/ + /e/ y /o/ + /o/ son res-

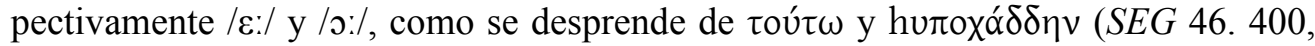
Esparta, 400- 375 a.C.), inscritos ya en alfabeto milesio. ${ }^{17}$ Con anterioridad, únicamente existen las grafías epicóricas $<\mathrm{E}>$ (cf. $\alpha \theta \rho \varepsilon \hat{\varepsilon} v, C E G$ 1.377, Esparta ca. 500-475?

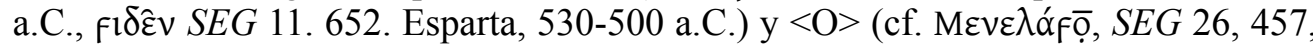
Esparta, ca. 675-650 a.C.; $\triangle \varepsilon \xi i ́ \pi \overline{0}, C E G$ 1.369, Delfos < Laconia, ¿ca. 600-550 a.C.?;

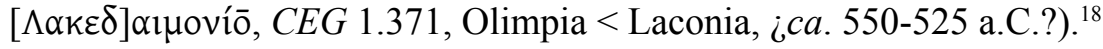

\subsubsection{Consonantismo}

El dialecto laconio es conservador en cuanto al tratamiento de ${ }^{*} w$. Como en la

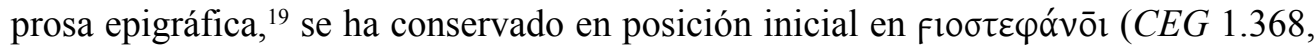

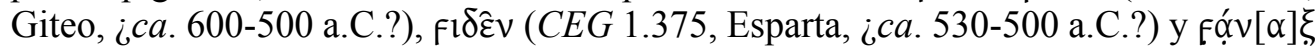

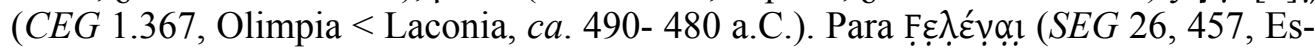
parta, $c a$. 675-650 a.C.) y Fekōßón̄ōı (CEG 1.370, Delfos < Laconia, s. vi a.C.), se han propuesto dos etimologías con * $w$ - o $* s w$ - a partir de los resultados con $\mathrm{f}$ - o con $/ \mathrm{h} / \mathrm{en}$ los diferentes dialectos $(\S 4.2 .1),{ }^{20}$ pero, en cualquier caso, se trata de las variantes locales. En posición intervocálica, la notación de /w/ es menos regular y su omisión

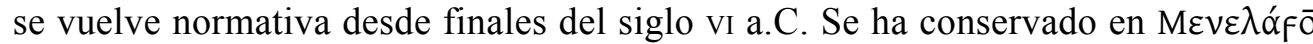
(SEG 26. 457, Esparta, ca. 675-650 a.C.) y hı $\lambda \vec{z} F$ Fo[1] (CEG 1.367, Olimpia, ca. 490480 a.C.), este último un siglo posterior; estas formas que contrastan con $\Delta \alpha \mu \bar{o} v \bar{o} v$

${ }^{14}$ Existe otro ejemplo métrico en 'E $\pi \alpha \underline{\alpha} \delta \rho i ́ \delta \bar{\alpha}$ (CEG 2.822, Gerontras, ¿s. IV a.C.?), cf. Striano (1989: 35).

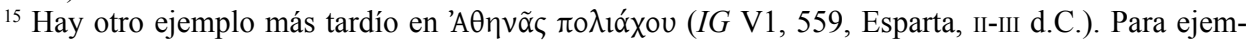

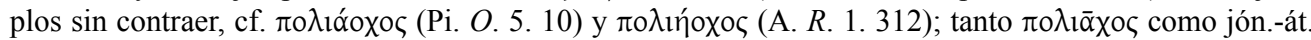

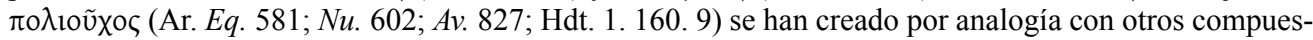
tos, cf. Buck (1955: § 167).

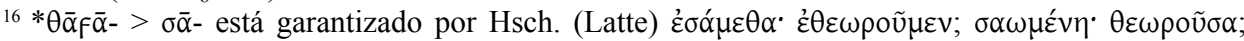

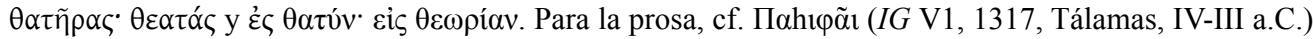

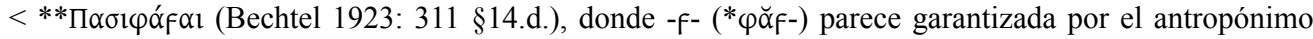
Фáßzvvoc (Syll. ${ }^{3}$ 422. 7, Delfos < Laconia, 266/262 a.C.).

${ }^{17}$ Cf. Striano (1989: 42 y 47).

${ }^{18}$ Respecto a los supuestos infinitivos breves en laconio, ninguno de los tres casos métricos presenta formas que confirmen su existencia: $<$ [ ]ANTA FI $\triangle \mathrm{EN}[$ ] $>$ (CEG 1.375, Esparta, ¿ca. 530-500 a.C.?), $<[$ ]PO $A \Theta P E N \Delta[$ ] $>(C E G$ 1.377.1, Esparta, ¿ca. 500-475 a.C.?) y $<\Lambda$ ABEN $>$ (CEG 2.820, Olimpia < Laconia, s. IV a.C.).

${ }^{19}$ Para el tratamiento de /w/ en los diferentes contextos fonéticos, cf. Striano (1989: 101-107).

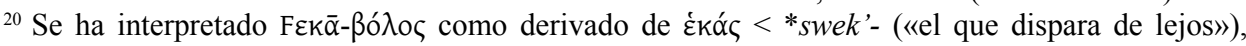
cf. Schwyzer (1939: 439 n. 8, $630 \S 4$ ), o de $\dot{\varepsilon} \kappa \omega v<*$ wek'- («el que dispara a voluntad»), cf. LSJ (s.v.

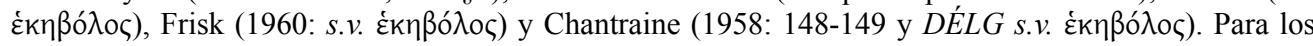
problemas que presenta la etimología de F̣ ç̣̂́ṿ̣̣, remitimos a Lanérès (2007) con la bibliografía anterior. 
(CEG 1.378, Esparta, s. v a.C.?) (<* $<\alpha \mu$ o-fóv $\alpha \xi), ~ \sigma \tilde{\alpha} \tau \rho o v$ y $\sigma \tilde{\alpha} \sigma \theta \alpha \mathrm{l}$ (SEG 46. 400, Esparta, 400-375 a.C.). No se conservan casos de notación de *(-)Cw- ya desde los primeros textos, como se aprecia en $\Delta \varepsilon \tilde{i} v 1[\zeta]$ (SEG 26. 457, Esparta, ca. 675-650 a.C.) $\left(<* \delta_{f \varepsilon l-}\right)$. Por otra parte, la pérdida de $* w$ en esta posición no provoca alargamiento de la vocal precedente; para las excepciones, cf. §4.2.1.

Existen tres ejemplos de notación $<\Delta(\Delta)>(<*(-) d j-)$ en ỏ $\pi 1 \delta(\delta)$ ó $\mu[\varepsilon v \circ \zeta](C E G 1.373$,

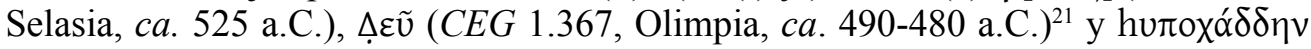

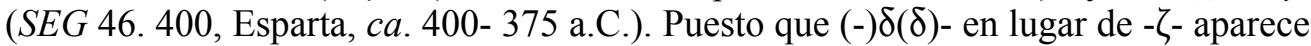
en las inscripciones métricas más antiguas y en la prosa arcaizante de época tardía, es la grafía dialectal, aunque a partir del siglo IV a.C. se generaliza $<\mathrm{Z}>$ en la prosa. ${ }^{22}$ La forma $\chi \alpha \rho \jmath \zeta o ́ \mu \varepsilon v[o \varsigma]$ (CEG 1.377, Esparta, ¿ca. 500-475 a.C.?) se explica porque aparece en un epigrama cuya lengua presenta una variedad poética de tipo dorio.

La poesía epigráfica laconia presenta el mismo tratamiento de la silbante que la prosa dialectal, como se observa en el debilitamiento de /s/ en posición final en țoĩ $(\lambda)$ $\Lambda \alpha \kappa \varepsilon \delta \alpha \iota \mu o v i ́ o[ı c] ~(C E G 1.367$, Olimpia < Laconia, $c a .490$ - 480 a.C.) y en la aspiración de /s/ intervocálica en vikóh $\alpha \varsigma$ ( $C E G$ 378, Esparta, ca. 450?-431 a.C.) y $\pi \tilde{\alpha} h ı v$ (CEG 2.821, Esparta, s. IV a.C.). Aunque $<\mathrm{H}>(=[\mathrm{h}])$ se detecta desde finales del s. VI a.C. y se generaliza a lo largo del s. v, en este periodo coexiste, igual que en la prosa,

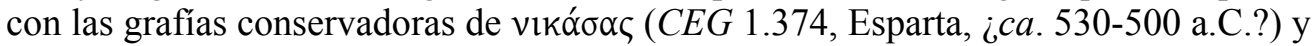
köpoløı (CEG 1.373, Selasia, ca. 525 a.C.). ${ }^{23}$

La aspiración inicial se nota hasta el s. IV en hop $\tilde{v}$ (CEG 2.821, Esparta, ¿s. IV

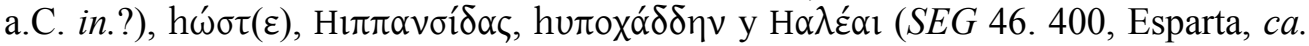
400-375 a.C.). ${ }^{24}$ En los tres últimos casos $/ \mathrm{h} /$ no es etimológica pero es pandialec-

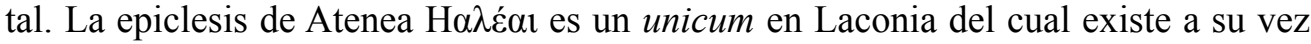
un correlato con aspiración en Arcadia que Dubois (1988: 47) explicó como una hi-

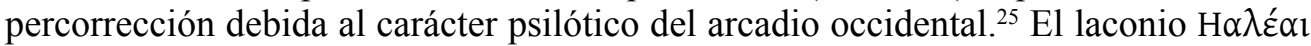
también puede ser una hipercorrección influida por el proceso $/ \mathrm{h} />/ \varnothing /$ del arcadio o bien porque el lapicida laconio consideró que el arcadio era psilótico y que la forma original era aspirada. ${ }^{26}$

${ }^{21}$ En $C E G$ y Striano (1989: 136) se sigue la lectura de Jeffery ( $L S A G$ p. 201 n. $\left.{ }^{\circ} 49\right)$ vs. Zẹ̃ de $I G$ V1, 1562a.

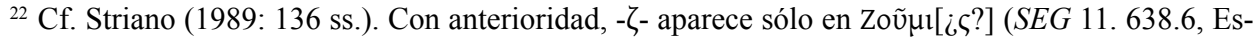
parta, 500-480 a.C.) cuya etimología es desconocida y podría ser además un nombre extranjero. Para la interpretación fonética de estas grafías, cf. Méndez Dosuna (1991-1993).

${ }^{23}$ Los primeros ejemplos $\sin / \mathrm{h} /$ son del siglo IV a.C. (Striano 1989: 160 y ss.). El debilitamiento no

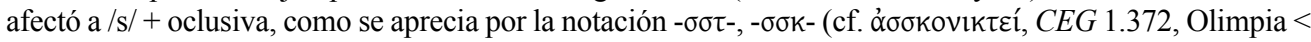
Laconia, ¿ca. 550-525 a.C.?). Para el debilitamiento de /s/ en laconio, cf. Alonso Déniz (2008: 37-182).

${ }^{24}$ Cf. Striano (1989: 175-179); para <HAMENKA $\Lambda$ H $>$ (SEG 46. 400), cf. Cassio (2000: 129-134) у Lanérès (2012: 717-719, 721-723).

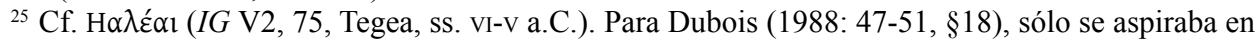
el sureste de Arcadia y esta fluctuación en la región habría generado formas hipercorrectas.

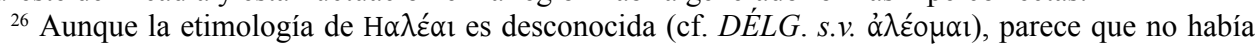
aspiración en la raíz (cf. $\dot{\alpha} \lambda \dot{\varepsilon}^{\prime}(\mathrm{f}) \mathrm{o} \mu \alpha \mathrm{l}<{ }^{*} h_{2} l e u$ - y posiblemente mic. $a$-re-wo 'A $\lambda \varepsilon^{\prime} f \omega v$ ) y que la psilosis se debió al jonio. Existe un homónimo $\alpha \lambda \hat{\varepsilon}^{\prime} \alpha$ («calor») cuyo origen suele ponerse en relación con *swelH-; su aspiración podría haber influido en la de $H \alpha \lambda \varepsilon ́ \alpha$. 
A partir del siglo Iv a.C. se fecha la espirantizatión de $/ \mathrm{t}^{\mathrm{h}} />/ \theta /(<\Sigma>)$. Se da en $S E G$

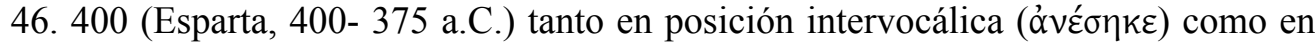
inicial $(\sigma \tilde{\alpha} \sigma \theta \alpha 1, \sigma \tilde{\alpha} \tau \rho \circ \nu)$ y precedida de nasal $(H \imath \pi \pi \alpha v \sigma i ́ \delta \alpha \varsigma) ;{ }^{27}$ con todo, en la prosa de este periodo todavía coexisten $\langle\Theta>$ y $\langle\Sigma>$ en una misma inscripción, tal como se

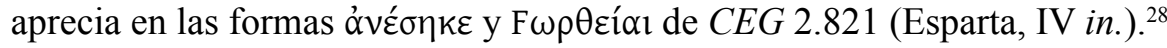

No es posible conocer el tipo de vocalismo resultante del tratamiento del grupo -Vns hasta la adopción del alfabeto milesio (cf. oủ $\delta \vec{\varepsilon} \varsigma, C E G$ 1.378, Esparta, $c a$. ¿450?431 a.C.), cuando los documentos epigráficos dialectales presentan $\langle\mathrm{H}>\mathrm{y}\langle\Omega>$, como en $\tau \hat{\omega} \varsigma$ $\delta \grave{\varepsilon} v \varepsilon ́ \omega \varsigma$ (SEG 46. 400, Esparta, 400- 375 a.C.), uno de los ejemplos más antiguos.

\subsection{Rasgos de los epigramas laconios atribuibles a la poesía épica}

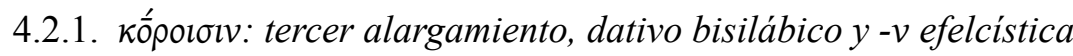

Dentro del corpus objeto de estudio, existen tres rasgos ajenos al dialecto laconio que se deben a la influencia de la lengua del epos: la desinencia de dativo plural bisilábica, el tercer alargamiento compensatorio y la $-v$ efelcística. Los tres aparecen concentrados en la secuencia $\Delta$ lò kốpoloıv en una inscripción del siglo vi a.C. (1).

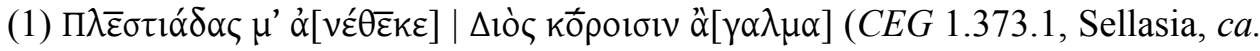
525 a.C.)

Se emplean por necesidades métricas debido a que la forma jónico-épica $\Delta$ iò kốpoloıv se ajusta al hexámetro ( - - - ` ) mejor que la forma local $\Delta$ iò kópoıৎ ( ( - ` -). Con todo, en la inscripción estos rasgos se combinan con otros de carácter epicórico cuando no existe ningún condicionamiento métrico. Como aquí, en la épica era habitual el alargamiento de $\kappa \bar{\rho} \rho-\left(<*\right.$ Ko $\left.f_{-}\right)$en esta fórmula y su aparición tras la cesura trocaica $(2 \mathrm{a}, 2 \mathrm{~b})$. Posteriormente, la tradición habría sido la encargada de autorizar y difundir esta fórmula en la poesía epigráfica $(2 \mathrm{c}) .^{29}$

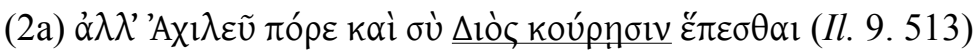

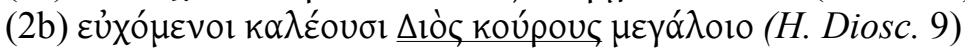

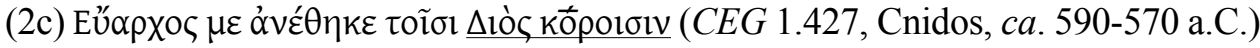

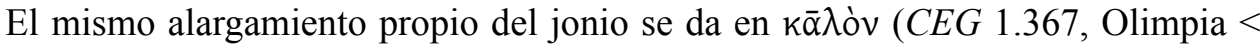
Laconia, ca. 490-480 a.C.) ( $\left.<{ }^{*} \kappa \alpha \lambda_{F}-\right)$. Es lo normal cuando forma parte de la secuen-

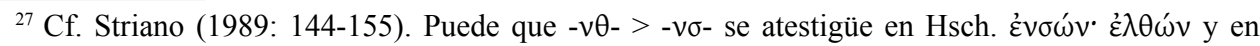

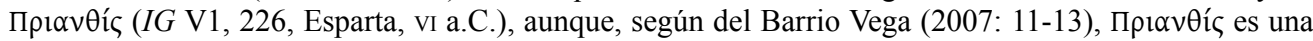

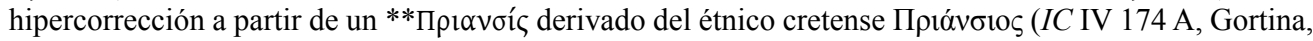
II a.C.).

${ }^{28}-\theta->-\sigma$ - parece no producirse tras /r/ y /s/ (Striano 1989: 147).

${ }^{29}$ Según Restelli (1951), el origen de la difusión del alargamiento de kov $\rho^{\circ}$ parte de la antigüedad y el prestigio de la fórmula en H. Diosc. (2b). 
cia dactílica $\kappa \alpha \lambda$ òv ${ }^{\prime} \not \gamma \alpha \lambda \mu \alpha$ a diferencia de las formas con sílaba breve ( $\left.\kappa \breve{\alpha} \lambda-\right)$ que aparecen cuando la inscripción está compuesta en yambos. ${ }^{30}$

También es épico el dativo -ololv (CEG 1.373, Selasia, ca. 525 a.C.) que contradice la desinencia monosilábica mayoritaria de la prosa (-olc); $;{ }^{31}$ en cuanto a la $-v$ efelcís-

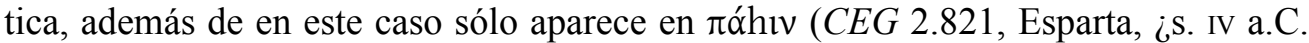
in.?), de modo que nunca afecta a los verbos en las inscripciones en verso laconias. Los datos métricos apuntan a que no es un rasgo dialectal porque se habría esperado su notación cuando el verbo aparece al final del verso, ${ }^{32} \mathrm{y}$, por otra parte, porque esta consonante habría evitado el hiato irregular de (3a) a juzgar por otros ejemplos como (3b). ${ }^{33}$ Parece, en cambio, que el carácter más reciente de esta fórmula podría estar detrás de la irregularidad prosódica. ${ }^{34}$

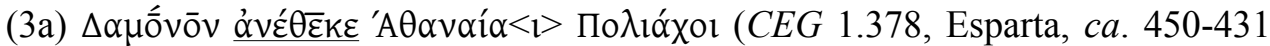
a.C.)

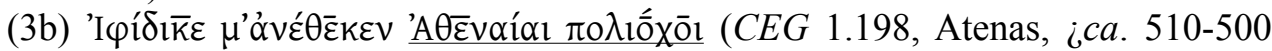
a.C.?)

Los poetas locales se han servido de otros mecanismos lingüísticos que desempeñan las mismas funciones que - $v$. Así, para resolver el hiato que se genera en la se-

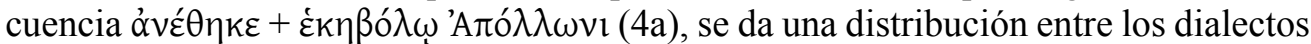
que como el laconio (4a) o el beocio (4b) mantienen F- en $f \varepsilon \kappa \alpha \beta o ́ \lambda \omega \omega$ igual que en la prosa, o los que, como el jonio $(4 \mathrm{c}, 4 \mathrm{~d})$ en el que ${ }^{*} w$ - ya ha desparecido, recurren a una

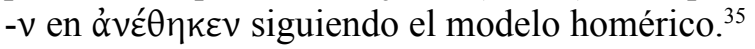

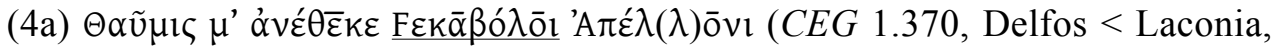
s. VI a.C.)

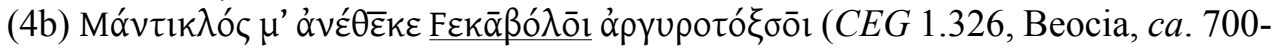
675 a.C.)

${ }^{30}$ Cf. $C E G 1.330$ (Beocia, ca. 500-475 a.C.), $C E G 1.447$ (Beocia, ca. 450-425) y $C E G 1.450$ (Lócride or., $c a .475$ a.C.).

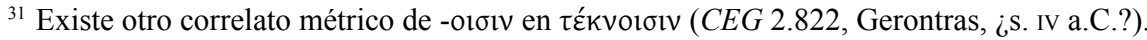

${ }^{32}$ Cf. $C E G$ 1.374.1 (Esparta, ¿ca. 530-500 a.C.?) o SEG 46. 400.1 (Esparta, 400-375 a.C.), donde además hay puntuación tras el verbo; - v habría sido contra metrum en SEG 26, 457 (Esparta, ca. 675650 a.C.), CEG 1.371 (Olimpia < Laconia, ¿ca. 550-525 a.C.?), CEG 372 (Olimpia < Laconia, ¿ca. 550525 a.C.?), y $C E G 1.370$ (Delfos < Laconia, s. vi a.C.).

${ }_{33}$ Para Striano (1989: 110-111), la - $v$ es dialectal debido a su presencia en dos apariciones de

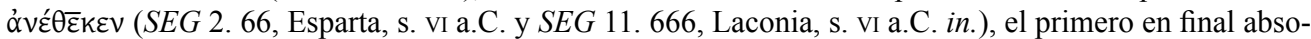
luto y el otro ante 'A $\theta \alpha v \alpha i ́ \alpha$. Sin embargo, abundan los contraejemplos sin -v (en final absoluto en $S E G$ 11.955, Laconia, VI ex.; IG 1565.2, Delfos < Laconia, 381/380 a.C. y ante 'A $\theta \alpha v \alpha i ́ \alpha$ en $I G$ 213.2, Esparta, s. v a.C.).

${ }^{34}$ La fórmula «Atenea protectora de la ciudad» se atestigua por primera vez en Píndaro (O. 5. 10), cf. también Ar. Eq. 581; Nu. 602; Au. 827; Hdt. 1. 160. 9; A. R. 1. 312. Para otra variante epigráfica, cf. $C E G 1.235 .1$ (Acrópolis, ¿ca. 500-480 a.C.?).

${ }^{35}$ Cf. Il. 1.14; Il. 1.110; Il. 16.513; Il. 23.872 o H. Ap. 177; H. Mus. et. Ap. 2. Una posible excepción podría verse en $I l .1 .21$, aunque los manuscritos ofrecen diferentes lecturas (cf. Chantraine 1958: 149). 


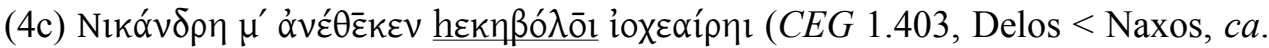
650 a.C.)

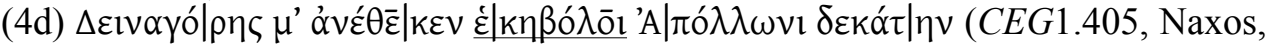
ca. 525 a.C.)

\subsubsection{Los alargamientos métricos}

Tampoco es propio del laconio el alargamiento de la primera sílaba de 'A $\bar{\varepsilon} \lambda(\lambda) \bar{o} v 1 .{ }^{36}$ Como en Homero, cuando el teónimo presenta cuatro sílabas en lugar de tres, debe escandirse - - - . El nombre a su vez se emplea dentro de una fórmula (4a) heredada de la épica en la que por necesidades métricas se han mantenido la escansión y la correptio epica aunque se ha adaptado a la fonética local (\$4.1.1).

\subsection{Rasgos atestiguados exclusivamente en los epigramas}

Se han conservado en las inscripciones métricas algunos adverbios con una anti-

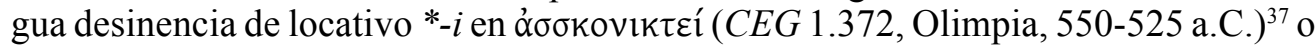
de instrumental en la correlación $\tau \alpha \cup \tau \tilde{\alpha} . . . ~ h \tilde{\alpha} \tau(\varepsilon)(C E G$ 1.378, Esparta, $c a .450$ ?-431

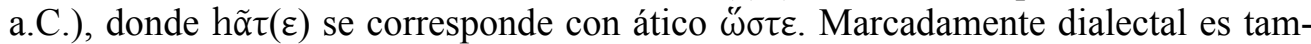

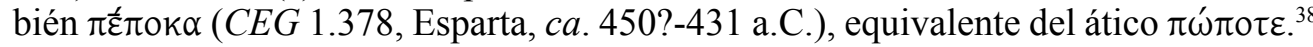

La poesía epigráfica ha conservado también léxico local en el dativo $\pi \varepsilon \rho \gamma u \tau \varepsilon ́ p o r \varsigma$

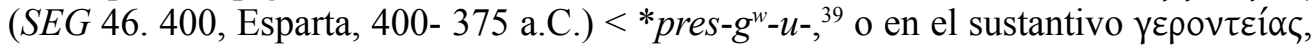

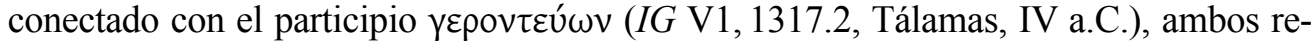

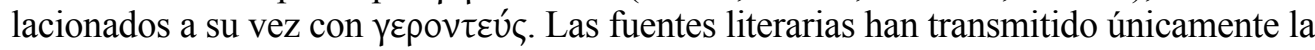
variante $\gamma \varepsilon \rho \circ v \tau i ́ \alpha(<\gamma \varepsilon \rho \circ \vee \tau-)$ que, en principio, presenta una derivación diferente de

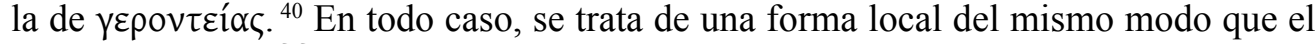
infinitivo huтoxá $\delta \delta \eta v ~(S E G ~ 46.400$, Esparta, 400- 375 a.C.), que es el único ejemplo

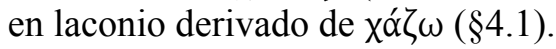

\section{RASGOS ORGÁNICOS E INORGÁNICOS EN LA POESÍA EPIGRÁFICA LACONIA}

En síntesis, los rasgos lingüísticos de las inscripciones métricas laconias anteriormente estudiados se resumen del siguiente modo:

${ }^{36}$ En el epos la primera sílaba del teónimo se alarga cuando es tetrasilábico; cf. Il. 1. 438 (misma fórmula) e Il. 4. 101, 119. Para este tipo de alargamientos de palabras antispásticas, cf. Chantraine (1958: 102).

${ }^{37}$ Se duda de si forma parte del hexámetro y de su relación con át. ớkovııí, «sin polvo».

${ }^{38}$ Para otras formas en laconio, cf. hó $\eta \eta$ ( $I G$ V1, 1155, Giteo, v a.C.) junto con $\pi \eta ́$ iok $\alpha$ (Theoc. 8. 34)

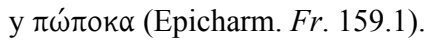

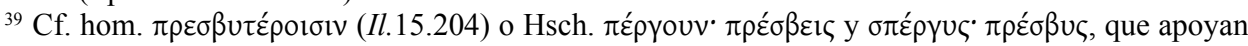
la evolución $\pi \rho \varepsilon \sigma \gamma v->\pi \rho \varepsilon \rho \gamma v->\pi \varepsilon \rho \gamma v-$, cf. Striano (2007: 458-459).

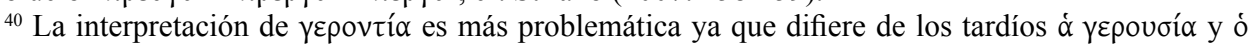

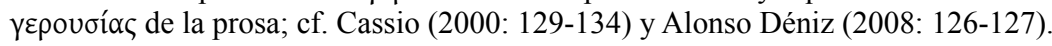




\begin{tabular}{|c|c|c|}
\hline $\begin{array}{c}\text { RASGOS COMPARTIDOS } \\
\text { POR LA POESÍA Y LA PROSA LOCALES }\end{array}$ & $\begin{array}{c}\text { RASGOS COMPARTIDOS } \\
\text { POR LA POESÍA EPIGRÁFICA Y ÉPICA }\end{array}$ & $\begin{array}{c}\text { RASGOS ATESTIGUADOS } \\
\text { SÓLO EN POESÍA EPIGRÁFICA }\end{array}$ \\
\hline 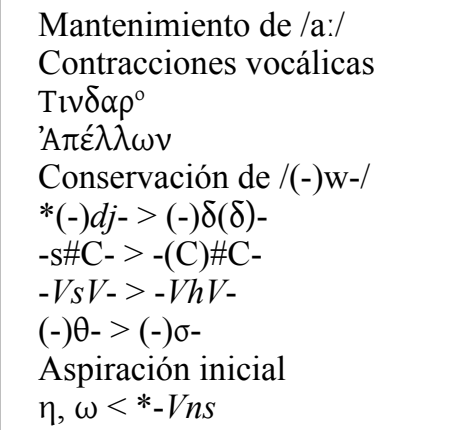 & $\begin{array}{l}-R V:-<*-R w V- \\
-\mathrm{ol \sigma} \sigma \\
-\nu \text { efelcística } \\
\text { alargamientos métricos }\end{array}$ & $\begin{array}{l}\text { Adverbios fosilizados } \\
\text { Léxico local }\end{array}$ \\
\hline
\end{tabular}

Tabla 1. Clasificación de los rasgos compartidos o propios de las inscripciones métricas laconias.

Si estos datos se clasifican atendiendo a su organicidad, es decir, a si se produce una oposición métrica entre la forma jónica y la epicórica, quedan distribuidos de la siguiente manera: ${ }^{41}$

\begin{tabular}{|c|c|c|}
\hline RASGOS INORGÁNICOS & \multicolumn{2}{|c|}{ RASGOS ORGÁNICOS } \\
\hline 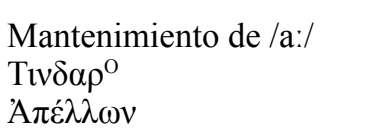 & Potenciales & $\begin{array}{l}\text { contracciones vocálicas } \\
\text { conservación de /(-)w-/ } \\
\text { adverbios fosilizados }\end{array}$ \\
\hline $\begin{array}{l}*(-) d j->(-) \delta(\delta)- \\
-\mathrm{s} \# \mathrm{C}->-(\mathrm{C}) \# \mathrm{C}- \\
-V s V->-V h V- \\
(-) \theta->(-) \sigma- \\
\text { Aspiración inicial } \\
\eta, \omega<*-V n s\end{array}$ & Plenamente orgánicos & $\begin{array}{l}-R V:-<*-R w V- \\
\text { dat. pl. -olol } \\
-\nu \text { efelcística } \\
\text { alargamientos métricos } \\
\text { correptio epica } \\
\text { léxico local }\end{array}$ \\
\hline
\end{tabular}

Tabla 2. Clasificación de los rasgos dialectales según el criterio métrico.

\subsection{Rasgos inorgánicos}

Todos los rasgos de carácter inorgánico son epicóricos y de carácter fonético. Esto se debe a que normalmente la fonética es el nivel gramatical menos afectado por la elección de las formas locales. Es significativo a su vez que todos estos rasgos sólo sean compartidos por la prosa y la poesía epigráficas (\$4.1). Si la lengua de los epigramas laconios hubiera sido la de la épica, se habría esperado la presencia de rasgos inorgánicos propios de la tradición jonia, o, dicho de otro modo, nunca se emplean

${ }^{41}$ Dentro de los rasgos orgánicos, se ha diferenciado entre los que pueden crear esta oposición (potencialmente orgánicos) o los que lo hacen de facto (plenamente orgánicos). 
rasgos jonios cuando equivalen métricamente a los epicóricos; más bien es al contrario, cuando no hay condicionamientos métricos, los poetas de esta región prefieren las formas propias de su dialecto.

\subsection{Rasgos orgánicos}

Se han conservado en estos textos tanto epicismos como dialectalismos irreductibles, lo que evidencia que ambos modelos son compatibles. Las formas épicas sólo se prefieren cuando las epicóricas no se ajustan al metro. Estas se concentran en dos epigramas: en $C E G 1.373$ (Selasia, ca. 525 a.C.) y en $C E G 2.821$ (Esparta, IV a.C.), donde coexisten con otros rasgos jonios (alargamiento $<*-R w V-$, dat. -olol, $-v$ efelcística), que coexisten a su vez con formas epicóricas. Junto con el alargamiento métrico (§4.2.2), todos estos jonismos afectan a la fonética y a la morfología, pero sobre todo a la prosodia, y son de un marcado carácter artificial. Las formas epicóricas orgánicas, por su parte, nunca aparecen en expresiones formulares y no se han visto influidas por un modelo literario (en tanto que variedad de prestigio). En definitiva, parece que ante la falta de un estándar poético-epigráfico, los poetas recurren a su dialecto. Estas formas dialectales se diferencian de las jonias en su fonética, pero también en su morfología y en el léxico.

\section{CONCLUSIONES}

Los datos de las inscripciones métricas laconias anteriores al ca. 400 a.C. muestran que la poesía epigráfica de la región está compuesta en dialecto local y con un componente épico motivado exclusivamente por factores métricos. En términos cuantitativos, el número de rasgos compartidos por las inscripciones laconias en verso y en prosa ( $\$ 4.1)$ es mucho mayor que los compartidos con la poesía jonia ( $\$ 4.2)$, mientras que en términos cualitativos los primeros son de carácter inorgánico y los segundos orgánicos. Cuando la métrica es indiferente, los poetas laconios emplean a su propio dialecto y cuando no lo es, recurren a formas jonias, si bien estas se reducen a contextos formulares. Fuera de las fórmulas heredadas de la tradición oral homérica no se detectan epicismos, sino rasgos locales que afectan a la fonética, a la morfología y al léxico. Una parte importante de estas fórmulas se adaptó a la fonética local si con ello no se alteraba la escansión. La creación de nuevas fórmulas a partir del modelo literario generó irregularidades prosódicas que muestran que cuando no existía un paradigma, se empleaba el dialecto laconio.

En definitiva, el análisis directo de los datos así como su clasificación a partir de criterios lingüísticos y métricos permite determinar que las conclusiones de Kock (1910) y particularmente de Alonso Déniz y Nieto Izquierdo (2009) para la poesía epigráfica argólica prehelenística se cumplen también en las inscripciones métricas laconias del mismo periodo: los poetas locales componen en su propio dialecto. 


\section{BiBLIOGRAFÍA}

Alonso DÉnIz, Alcorac (2008), Estudios sobre la aspiración de /s/ en los dialectos griegos del I milenio, Editorial Complutense. Madrid.

- (2014), «L'esprit du temps: koinè, dialecte et hyper-dialecte dans les inscriptions agonistiques d'Artemis Orthia à Sparte», en S. Minon (ed.) Diffusion de l'attique et expansion des koinai dans le Peloponnese et en Grece centrale: actes de la journee internationale de dialectologie grecque du 18 mars 2011, universite Paris-Ouest Nanterre, Ginebra: 141-168.

Alonso Déniz, Alcorac y Nieto IzQuierdo, Enrique (2009), «Dialecto local y dialecto épico en las inscripciones métricas de la Argólide», Minerva 22: 83-105.

Del Barrio Vega, M. ${ }^{a}$ Luisa (2007), «A propos de quelques formes du laconien et du messenien», en I. Hajnal (ed.), Die Altgriechischen Dialekte. Wesen und Werden. Akten des Kolloquiums Freie Universität Berlin 19-22 September 2001, Innsbruck: 1-17.

Bechtel, Friedrich (1923), Die griechischen Dialekte 2. Die westgriechischen dialekte. Berlin. BING, Peter y Bruss, John S. (2007), A Brill's Companion to Hellenistic Epigram. Leiden.

Buck, Carl D. (1912), «A New Epigram From Thessaly BCH (1911) XXXV 239», CPh 7: 351-353.

- (1924), "A Question of Dialect Mixture in the Greek epigram», Antidoron Festschrift: Jacob Wackermagel zur Vollendung des 70, Göttingen: 32-136.

CAssio, Albio C. (2000), «Un epigramma votivo per Atena Alea», RFIC 128 (2): 129-134.

Chantraine, Pierre (1958), Grammaire homérique. Tome I: Phonétique et morphologie. Paris. $D E ́ L G=$ ChantraIne, Pierre et al. (2009), Dictionnaire étymologique de la langue grecque: histoire des mots. Paris.

Dubois, Laurent (1988), Recherches sur les dialecte arcadien. Louvain-la-Neuve.

FrISK, Hjalmar (1960-1972), Griechisches etymologisches Wörterbuch I-III. Heidelberg.

CEG 1= HANSEN, Peter A. (1983), Carmina Epigraphica Graeca saeculorum VII-V a.Chr. N. Berlin, New York.

CEG 2= HAnsen, Peter A. (1989), Carmina Epigraphica Graeca saeculi vi a. Chr. N. Berlin, New York.

Hoffmann, Otto, Debruner, Albert, Scherer, Wilhelm (1973), Geschichte der griechischen Sprache. Berlin.

KACZKO, Sara (2009), «From Stone to Parchment: Epigraphic and Literary Transmission of Some Greek Epigrams», Trends in Classics 1/1: 90-117.

Kock, Bertrand (1910), De epigrammatum Graecorum dialectis. Göttingen.

Kretschmer, Paul (1915), «Literaturbericht für das Jahr 1912», Glotta 6: 273-311.

LANÉRÈs, Nicole (2007), «L'Harpax de Thérapné ou le digamma d'Hélène», Phōnēs charaktēr ethnikos: actes du Ve Congrès international de dialectologie grecque (Athènes 28-30 septembre 2006): 236-269.

- (2012), «La dédicace du «trône» d'Aléa $S E G$ 46, 400, nouvelle lecture», REG 125: 715-725. $L S A G=$ JEFFERY, Lilian H. (1990) ${ }^{2}$, The Local Scripts of Archaic Greece. Oxford.

MÉndez DosunA, Julián (1991-1993), «On $<\mathrm{Z}>$ for $<\Delta>$ in Greek dialectal inscriptions», Die Sprache 35: 82-114.

Mickey, Katherine (1981a), Studies in the Greek Dialects and the Language of Greek Verse Inscriptions, Tesis doctoral inédita. Oxford. 
Mickey, Katherine (1981b), «Dialect Consciousness and Literary Language: An Example from Ancient Greek», TPhS 79: 35-66.

Palumbo, Bruna (1987), «Differenze dialettali e stilistiche nella storia dell'epigramma greco», en G. Bolognesi y V. Pisani (eds.), Linguistica e filologia. Atti del VII Convegno Internazionale di Linguistici, Milano 12-14/09/1984, Brescia: 429-434.

Puelma, Mario (1997), «Epigramma: osservazioni sulla storia di un termine greco-latino», Maia 49.2: 189-123.

Restelli, Giuseppe (1951), «La forma $\Delta$ lóбkoupoı nelle più importanti epigrafi greche», RFIC 29: 246-257.

Schwyzer, Eduard (1939), Griechische Grammatik. Allgemeiner Teil, Lautlehre, Wortbildung, Flexion. München.

Striano = Striano Corrochano, Araceli (1989), El dialecto laconio. Gramática y estudio dialectal, Tesis doctoral inédita. Universidad Autónoma de Madrid.

Striano Corrochano, Araceli (2007), «À propos d'une nouvelle inscription laconienne», en I Hajnal (ed.), Die Altgriechischen Dialekte. Wesen und Werden. Akten des Kolloquiums Freie Universität Berlin 19-22 September 2001, Innsbruck: 449-462.

TIBERI, Luca (1996), «Epigrammi tramandati in versione epigráfica e in versione letteraria», Lexis 14: 71-85.

Thumb, Albert y KIECKers, Ernst (1932), Handbuch der griechischen Dialekte I. Heidelberg. 